\title{
CFD-MRI: A coupled measurement and simulation approach for accurate fluid flow characterisation and domain identification
}

\author{
Fabian Klemens ${ }^{\mathrm{a}, \mathrm{b}, *}$, Sebastian Schuhmann ${ }^{\mathrm{c}}$, Gisela Guthausen ${ }^{\mathrm{c}}$, Gudrun Thäter ${ }^{\mathrm{a}, \mathrm{b}}$, \\ Mathias J. Krause ${ }^{a, b, c}$ \\ a Lattice Boltzmann Research Group (LBRG), Germany \\ ${ }^{\mathrm{b}}$ Institute for Applied and Numerical Mathematics (IANM), Karlsruhe Institute of Technology (KIT), Karlsruhe 76131, Germany \\ ' Institute for Mechanical Process Engineering and Mechanics (MVM), Karlsruhe Institute of Technology (KIT), Karlsruhe 76131, Germany
}

Keywords:

Lattice Boltzmann method

Simulation

MRI

Optimisation

Fluid characterisation

Domain identification

\begin{abstract}
A B S T R A C T
This article presents the coupling of magnetic resonance imaging (MRI) measurements and computational fluid dynamics (CFD) for accurate characterisation of fluid flow and identification of flow domains. Currently, MRI measurements are averaged over time and space, assuming a certain smoothness of the velocity and pressure space. However, a possible solution of a fluid problem must fulfil the Navier-Stokes equations, which sets up a condition that is much more restrictive than the usual smoothness assumptions in e.g. curve fitting. The novel CFD-MRI method uses this insight to reduce the statistical noise and to identify finer structures of the underlying domain. The problem is formulated as a distributed control problem which minimises the distance between measured and simulated flow field. Thereby, the simulated flow field is the solution of a parametrised porous media BGK-Boltzmann equation which approaches a homogenised Navier-Stokes equation in the hydrodynamic limit. The parameters represent the porosity distributed in the domain which yields a domain and a fluid flow that fits best to the measured data. This enables the method they locate an obstacle and the flow field from limited $2 D$ spatially resolved MRI data with one velocity component. The problem is solved with an adjoint lattice Boltzmann method (ALBM) using the open source software OpenLB ${ }^{1}$.
\end{abstract}

\section{Introduction}

Improving magnetic resonance imaging (MRI), in particular flow phase contrast MRI (flow MRI), which can depict fluid flows in complex geometries, is a very important task. In medical applications, improved accuracy can help diagnose severe health issues, e.g. by locating artery narrowing that indicates the risk of a heart attack. In technical applications, it can help to identify problems, for example in filtration processes, in fluidic bypass solution or in refining steps in crude oil industry.

MRI is known to be a versatile tool to investigate the interesting object non-destructively and non-invasively with a low energy input compared to e.g. x-ray tomography [1-3]. Structural and functional images as well as flow fields are known to reveal indepth insight. As often time-dependent processes are investigated or patients and animals may stay only a limited time in a magnet,

* Corresponding author at: Institute for Applied and Numerical Mathematics (IANM), Karlsruhe Institute of Technology (KIT), Karlsruhe, 76131, Germany.

E-mail address: fabian.klemens@kit.edu (F. Klemens).

1 http://www.openlb.net the measurement time is limited. The MRI community is therefore searching to improve the time resolution, i.e. to shorten the time required to perform an MRI experiment, while retaining the spatial resolution and the level of information. Current approaches are the use of higher magnetic fields and the application of compressed sensing algorithms.

A different way to improve flow MRI is to combine measurement and simulation, here called CFD-MRI method. This coupling can be achieved by formulating a restricted optimisation problem, called fluid flow domain identification problems, which minimises the distance of measured and simulated flow fields. The optimisation problem thereby only allows solutions which fulfil corresponding physical properties and equations, here the Navier-Stokes equations for fluid flow problems. This promises to lead to a considerably more accurate characterisation of fluid flows and flow domains in complex geometries.

The main aim of this manuscript is to introduce, realise and validate the proposed CFD-MRI method for the coupling of measured data and simulation using the adjoint lattice Boltzmann method (ALBM) for solving fluid flow domain identification problems $[4,5]$. The method is used to locate an object and the flow field using 
only limited $2 D$ spatially resolved MRI data with one velocity component.

The combination of MRI data and CFD simulation is often used in medical applications, where the MR images are used to obtain velocity profiles or the reconstruction of geometries in patient data [6-9]. However, to the knowledge of the authors there has never been a coupling of simulation and measurement data as input for a topology optimisation problem in order to improve the quality of the data.

In the field of computational fluid dynamics (CFD), the lattice Boltzmann method (LBM) is a well established method for solving incompressible Navier-Stokes equations [10-13]. Thereby, the big advantages of LBM are its easy implementation due to the simple core algorithm and its local computations, making it a very powerful numerical tool in high performance simulation of fluid flow problems [14,15]. For adjoint based optimisation in LBM there are two main methods: The first-discretise-then-optimise, where the adjoint equations are derived from the discrete lattice Boltzmann equations, and the first-optimise-then-discretise approach, where the adjoints are derived from the continuous BGKBoltzmann equation and discretised afterwards. The first method was proposed by Tekitek et al. [16] for parameter identification, and was later used for topology optimisation by Liu et al. [17] or Nørgaard et al. [18]. The latter method, called adjoint lattice Boltzmann method (ALBM), was proposed by Krause et al. [19] showing its effectiveness for parallel implementation and high performance. ALBM is nowadays most often used for topology optimisation, e.g. by Yaji et al. [20,21]. The first to use LBM for topology optimisation were Pingen et al. [22] using a porous media model. Based on this, Krause et al. [4,5] have proposed an extension to the ALBM for the determination of flow areas and flow dynamics. Thereby, porosity is the control variable of an optimisation problem that minimises the resulting velocity distributions to those of a measured one. The optimisation problem is solved with a gradient-based method, e.g. L-BFGS [23], where the result is the distribution of porosity inside the domain. The ALBM was validated for domain identification problems for different and increasingly complex objects and with complete and partially available artificial data (cf. $[4,5])$. However, no validation of the algorithm with measurement data was performed, which is one of the formulated aims of this manuscript.

The manuscript is structured as follows: Section 2 introduces the CFD-MRI method. As simulation method, a parametrised porous media BGK-Boltzmann method for the flow through porous media will be presented. Next, the flow MRI to obtain the fluid flow data in physical experiments is introduced. Finally, simulation and measurement are combined to an optimisation problem, the fluid flow domain identification problem, which is solved using an adjoint lattice Boltzmann method. Section 3 contains the conducted experiments and validation of the method. First the MRI experiment and its results are described and then the resulting measurement data is used for the CFD-MRI method in the numerical experiments.

\section{CFD-MRI method}

The general idea of the CFD-MRI method is to use MRI data as initial values for the simulation. That is, the geometry, represented as porosity distribution, and physical quantities like maximum velocity or velocity profile for example. The MRI data and the simulation are then combined to an optimisation problem, where the difference of simulated and measured flow field is minimised. This leads to a new distribution of porosity in the domain and is repeated until the flow fields coincide. The result is then a distribution of porosity and velocity that matches the data.

\subsection{Parametrised fluid flow simulation}

For the simulation of incompressible Newtonian fluid flows a LBM is chosen. A porous media model is used here, since the method needs to be able to change the topology of the domain. This is based on the idea of Pingen et al. [22], who used a porosity function to scale the velocity in the equilibrium distribution function, which recovers the Brinkman equations for flow through porous media. The porous media BGK Boltzmann equation introduced by Krause et al. $[4,5]$ is defined as

$h^{2} \frac{d}{d t} f+\frac{1}{3 v}\left(f-f_{d}^{e q}\right)=0$,

where $f=f(t, \mathbf{r}, \mathbf{c})$ is the particle distribution function, with time $t \in I=\left[t_{0}, t_{1}\right) \subseteq \mathbb{R}_{\geq 0}$, position $\mathbf{r} \in \Omega \subseteq \mathbb{R}^{d}$, velocity $\mathbf{c} \in \mathbb{R}^{d}$, model parameter $h \in \mathbb{R}_{>0}$ and kinematic viscosity $v \in \mathbb{R}$. The equilibrium distribution function $f_{d}^{e q}(\rho, \mathbf{u})$ is a function of the macroscopic density $\rho$ and velocity $\mathbf{u}$ and depends on the porosity function $d: \Omega \rightarrow[0,1]$. For every $\mathbf{r} \in \Omega$ the porosity can then be defined as $d(\mathbf{r})=0$ for solid, $d(\mathbf{r})=1$ for fluid and $d(\mathbf{r}) \in(0,1)$ for porous materials.

For discretisation, we use the lattice Boltzmann method (LBM), which leads to the lattice Boltzmann equation (LBE) defined as

$f_{i}\left(\mathbf{x}+\mathbf{c}_{i} h^{2}, t+h^{2}\right)-f_{i}(\mathbf{x}, t)=-\frac{1}{\tau}\left(f_{i}(\mathbf{x}, t)-f_{i, d_{h}}^{\text {eq }}(\rho, \mathbf{u})\right)$.

Here, $f_{i}: \Omega_{h} \times I_{h} \rightarrow \mathbb{R}^{+}$for $i=0, \ldots, q-1$ are the distribution functions with $\Omega_{h} \subset \Omega$ the lattice, $I_{h}:=\left\{t \mid t=k h^{2}, k=0, \ldots, n_{k}\right\}$ the discrete time interval and $h$ now the discretisation parameter. The relaxation time $\tau$ is associated with the kinematic viscosity $v$ by the relation $\tau=3 v+1 / 2$. The discrete velocities $\mathbf{c}_{i} \in \mathbb{R}^{d}(i=$ $0, \ldots, q-1)$ are chosen such that $\mathbf{c}_{i}+\mathbf{x} h^{2} \in \Omega_{h}$, where the discretisation model is defined as DdQq. Based on Spaid and Phelan [24], Krause et al. [4,5] proposed a grid independent function $d_{h}: \Omega_{h} \rightarrow[0,1]$ defined as

$d_{h}:=d_{h}(\mathbf{x})=1-h^{2} \frac{\nu \tau}{K}$

with permeability $K$. Here, $d_{h}$ is defined as the lattice porosity, which is related to physical porosity in the sense that a point $\vec{x} \in \Omega_{h}$ is regarded as solid for $d_{h}=0$ (zero permeability), as fluid for $d_{h}=1$ (infinite permeability) and occupied by a porousmedium for $d_{h} \in(0,1)$ [22]. The porous media equilibrium distribution function $f_{i, d_{h}}^{e q}(i=0, \ldots, q-1)$ is then defined as

$$
\begin{aligned}
f_{i, d_{h}}^{e q}(\rho, \mathbf{u})= & w_{i} \rho\left(1+3 h^{2}\left(\mathbf{c}_{i} \cdot d_{h} \mathbf{u}\right)-\frac{3}{2} h^{2}\left(d_{h} \mathbf{u} \cdot d_{h} \mathbf{u}\right)\right. \\
& \left.+\frac{9}{2} h^{4}\left(\mathbf{c}_{i} \cdot d_{h} \mathbf{u}\right)^{2}\right) .
\end{aligned}
$$

For $D 3 Q 19$ the weights $w_{i}$ are defined as $w_{0}=1 / 9 w$, $w_{i}=1 / 18 w \quad(i=1, \ldots, 6), \quad w_{i}=1 / 36 w \quad(i=7, \ldots, 18) \quad$ and $w=2 / 3 \pi^{3 / 2} h^{-3} \exp \left(3 / 2 \mathbf{c}_{i}^{2}\right)$. This method is also a special case of the homogenised lattice Boltzmann method (HLBM) [25]. The macroscopic quantities velocity $\mathbf{u}: \Omega_{h} \times I_{h} \rightarrow \mathbb{R}^{d}$ and density $\rho: \Omega_{h} \times I_{h} \rightarrow \mathbb{R}$ can be computed as moments of the distribution function as follows:

$$
\rho(\mathbf{x}, t)=\sum_{i=0}^{q-1} f_{i}(\mathbf{x}, t), \quad \rho \mathbf{u}(\mathbf{x}, t)=\sum_{i=0}^{q-1} \mathbf{c}_{i} f_{i}(\mathbf{x}, t) .
$$

\subsection{Flow MRI}

Flow fields can be measured by MRI by exploring the nuclear magnetisation's phase and the impact of coherent motion of spins onto this nuclear magnetic resonance (NMR) quantity [1-3]. It is well known that by encoding and decoding of the magnetisation's phase at a later time, velocity can be calculated spatially resolved. 


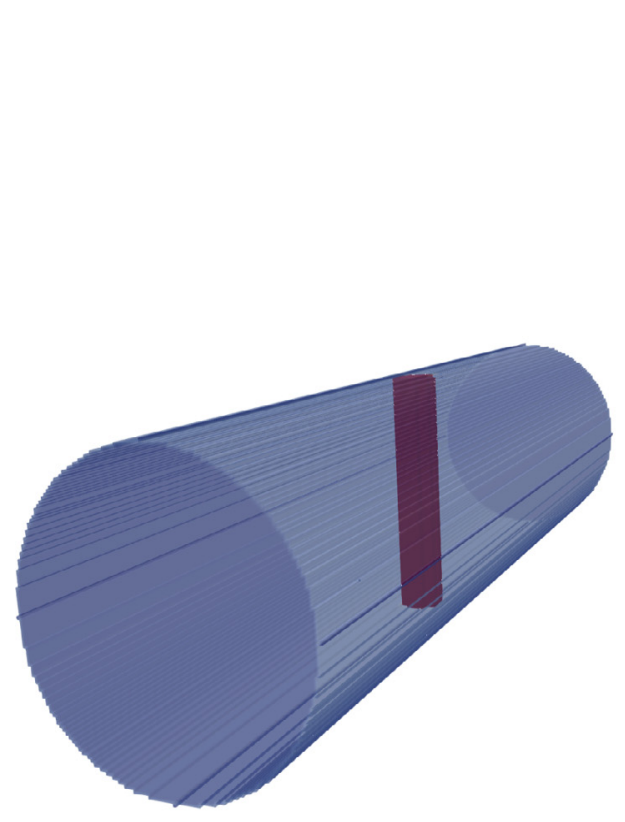

(a) Schematic: Geometry of the Domain.

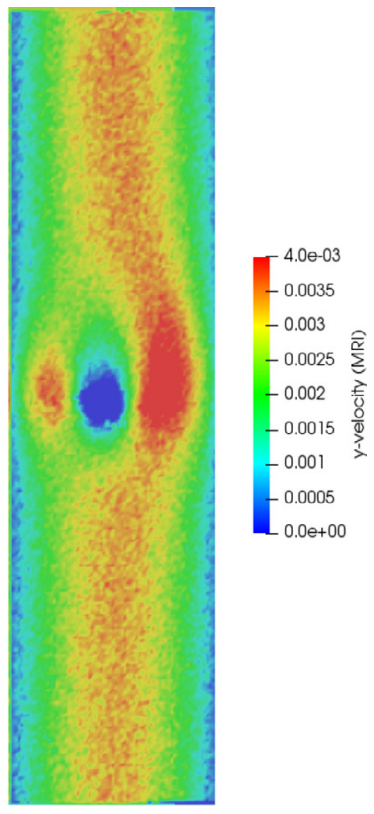

(b) MRI data.

Fig. 1. MRI experiment. Left, the schematic setup of the experiment. A cylindrical object (red) inside a tube of length $0.0255 \mathrm{~m}$ and radius of $0.004 \mathrm{~m}$. Right, the results of the MRI experiment. The data is the $y$-velocity component of the flow in the $x-y$ plane located in the middle of the tube. (For interpretation of the references to colour in this figure legend, the reader is referred to the web version of this article.)

Several experimental realisations are known, first the Fourier velocity imaging which results in velocity maps and additionally in maps of velocity amplitude. A much faster approach is the FlowPC approach where only one flow phase encoding gradient amplitude is employed rather than a sampling of the complete inverse, Fourier conjugated space. This approach usually leads to a time reduction of a factor of $8-32$ in $2 D$ spatially resolved flow experiments. It can be realised as gradient echo sequence or as spin echo sequence. Data in this publication were acquired by the flow encoding gradient echo sequence. As the aim of the investigation was to show the capability of the combined CFD-MRI approach, the measurement parameters were chosen to minimise the measurement time, i.e. the spatial resolution as well as the signal-to-noise ratio were at the lower limit of acceptance.

\subsection{Fluid flow characterisation and domain identification}

For the fluid flow characterisation we use an optimisation approach for solving domain identification problems [4,5]. This is done by introducing a goal function defined as

$J(f, \alpha)=\frac{1}{2} \int_{\Omega_{\jmath}}\left(\mathbf{u}_{f}-\mathbf{u}^{*}\right)^{2} d \mathbf{r}$,

which calculates the difference between a given flow field $\mathbf{u}^{*}$ and a simulated flow field $\mathbf{u}_{f}$ inside an objective domain $\Omega_{J} \subset \Omega \subset \mathbb{R}^{d}$. The goal function is minimised using a control $\alpha$, where $\alpha$ changes the lattice porosity inside the objective domain by a projection $B$ : $\alpha \mapsto B \alpha:=d$. Thereby, only states $f$ are admissible which fulfil the porous media BGK Boltzmann equation (1), linking control and state. This is the side condition $\mathbf{G}(f, \alpha)$ of the optimisation problem and here defined as

$\mathbf{G}(f(\alpha), \alpha):=h^{2} \frac{d}{d t} f+\frac{1}{3 v}\left(f-f_{B \alpha}^{e q}\right)$.

Note that in this method a steady state solution is assumed.

In order to find the optimal control a quasi-Newton method is used (e.g. L-BFGS [23]), for which the total derivative of the goal function with respect to the control is needed. In $[4,5]$ this total derivative is found to be

$$
\frac{d}{d \alpha} J(f(\alpha), \alpha)=u_{f} \tau \int_{\mathbb{R}^{d}} \varphi 3 h^{2}\left(v-B \alpha \mathbf{u}_{f}\right) f_{B \alpha}^{e q} d v+\frac{\partial}{\partial f} J
$$

where $\varphi$ is determined by solving an adjoint porous media BGKBoltzmann equation

$\frac{d}{d t} \varphi-\frac{1}{3 v}\left(\varphi-\varphi_{B \alpha}^{e q}\right)+\frac{\partial}{\partial f} J=0$,

with adjoint equilibrium distribution function $\varphi_{B \alpha}^{e q}$, which depends on the macroscopic quantities of the porous media BGK-Boltzmann equation (1).

Using the adjoint lattice Boltzmann method (ALBM) [19] the discrete adjoint porous media lattice Boltzmann equation is found to be

$$
\begin{aligned}
\varphi_{j}\left(\mathbf{x}-\mathbf{c}_{j} h^{2}, t-h^{2}\right)-\varphi_{j}(\mathbf{x}, t)= & -\frac{1}{\tau}\left(\varphi_{j}(\mathbf{x}, t)-\varphi_{j, B \alpha}^{e q}(\mathbf{x}, t)\right) \\
& +\frac{3 v}{\tau} h^{2} d J_{j}
\end{aligned}
$$

with $d J_{j}=-\frac{\left(\mathbf{u}^{*}-\mathbf{u}\right)\left(\mathbf{c}_{j}-\mathbf{u}^{*}\right)}{\rho}$ and discrete adjoint equilibrium distribution function

$\varphi_{j, B \alpha}^{e q}=\sum_{i=0}^{q-1} \varphi_{i} \frac{\left(3 B \alpha\left(B \alpha h \mathbf{u}-\mathbf{c}_{j}\right)\left(h \mathbf{u}-\mathbf{c}_{i}\right)+1\right)}{\rho} f_{i, B \alpha}^{e q}$.

Note that the adjoint equilibrium distribution function depends on the LBE (2) and its moments. The discrete adjoint porous media lattice Boltzmann equation (10) is also very similar to the porous media lattice Boltzmann equation (2), leading itself to the same high efficient parallelisation. This makes it possible to calculate the derivatives and the objective function, required for the quasi-Newton method, very fast. By using an efficient optimisation method such as L-BFGS, the performance can be further improved. For the numerical experiments in Section 3 a L-BFGS method was implemented in the OpenLB framework. 


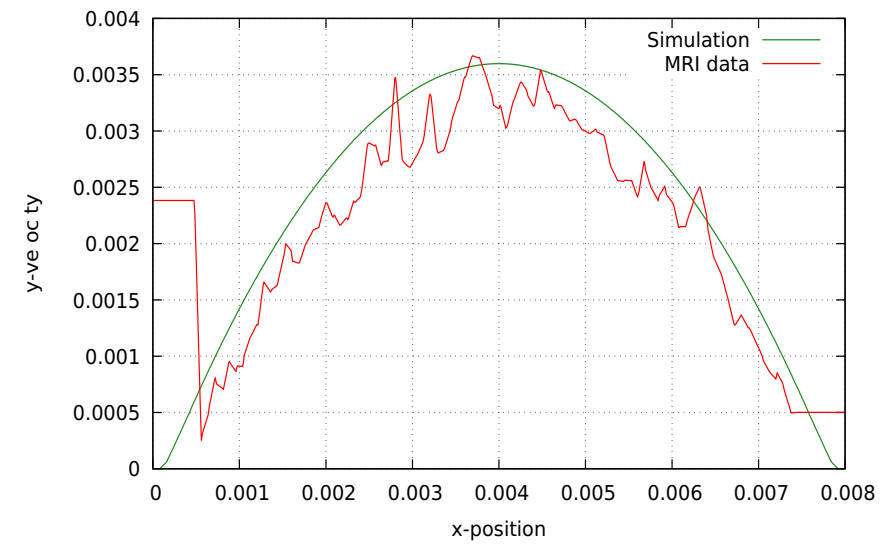

Fig. 2. Poiseuille profile. Here the velocity profile of MRI data (red) and simulation (green) along a line at $(x, 0.00025,0.004)^{T}, x \in[0,0.008]$ is shown. (For interpretation of the references to colour in this figure legend, the reader is referred to the web version of this article.)

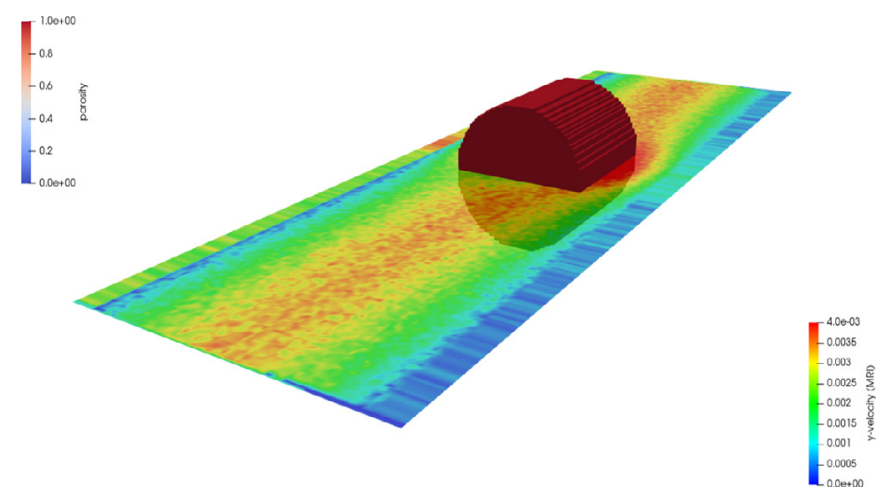

Fig. 3. Design Domain. Start of the design domain (red) corresponding where the cylinder is assumed in the MRI data (plane). (For interpretation of the references to colour in this figure legend, the reader is referred to the web version of this article.)

\section{Validation and application}

In this section, the experimental and numerical setup is described, and the results for the CFD-MRI method are discussed.

\subsection{MRI experimental setup}

The experiments were performed on a $200 \mathrm{MHz}$ super wide bore MRI instrument (Bruker BioSpin GmbH, Rheinstetten) equipped with an Avance HDIII console. The probe used for the experiments was a MICWB 40 with a $10 \mathrm{~mm}$ birdcage. Gradients up to $1 \mathrm{~T} / \mathrm{m}$ for the imaging and flow encoding were available. As the aim was to condense information into fast experiments, only $2 D$ spatially resolved flow images were acquired in a measurement time of several minutes via Flow map in the Bruker Paravision 6.0 software. Data processing was performed by in house written Matlab scripts.

A model was realised for the flow measurements. A straight, stiff tube was modified by placing an obstacle, here a cylindrical specimen (see Fig. 1(a)). This stiff tube was connected via flexible tubes to a chromatographic pump with minimal pulsation to realise a stationary flow field in the model. As flowing liquid, isopropanol was chosen. The MRI experimental result is shown in Fig. 1(b).

\subsection{Simulation setup}

For the numerical experiments, the tube is assumed to be a cylinder with length of $0.0255 \mathrm{~m}$ and radius of $0.004 \mathrm{~m}$, which

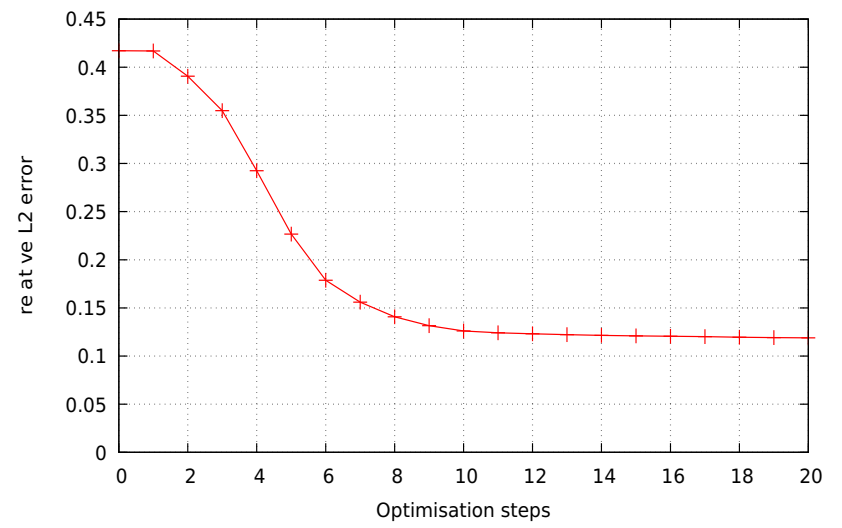

Fig. 4. Objective plot. Here, the relative $L 2$ error $\|\cdot\|_{L_{\text {rel }}^{2}}$ over optimisation steps is shown. The error starts at $41.72 \%$ and reaches $11.83 \%$, which is at the low end of the measurement error.

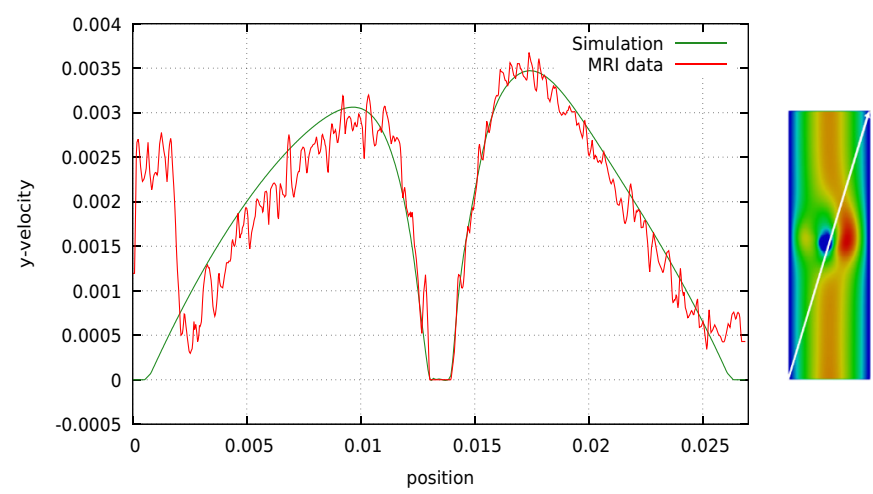

Fig. 5. Velocity profile: across the domain. Comparison of velocity profile along a line from bottom left to top right of the plane (white line in right figure). The experimental data is shown in red and the simulation result in green. Here, a significant reduction in measurement noise can be seen. (For interpretation of the references to colour in this figure legend, the reader is referred to the web version of this article.)

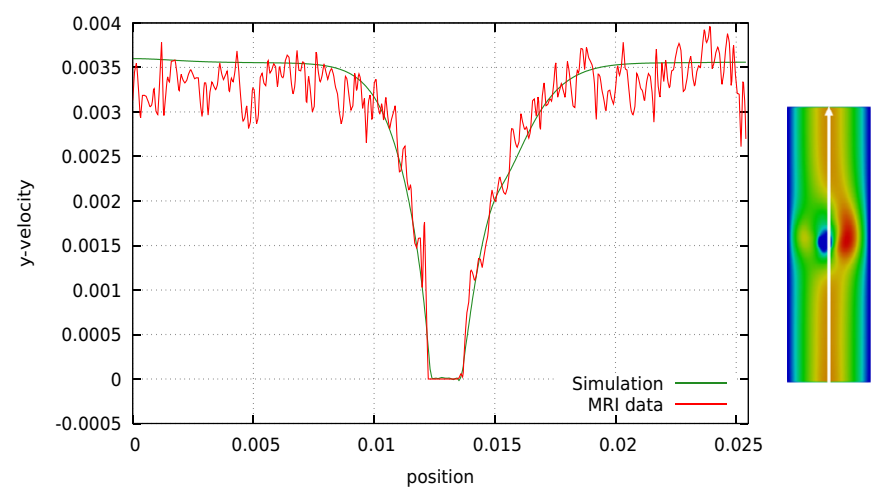

Fig. 6. Velocity profile: $y$-axis. Comparison of velocity profiles along $y$-axis (white line in right figure), with a significant reduction of the measurement noise. The experimental data is shown in red and the simulation result in green. (For interpretation of the references to colour in this figure legend, the reader is referred to the web version of this article.)

matches the MRI experiment (cf. Section 3.1). The domain is discretised using a resolution of $100 \times 256 \times 100$ cells, resulting in around 2.5 million lattice nodes total. The MRI data consists of $128 \times 256$ data points, where the outer approximately 50 data points are only noise. A bilinear interpolation is used to provide the velocity information of the MRI data on the lattice. Therefore, the method is independent of the resolution of the measurement data. A velocity boundary condition is set at the inflow, with Poiseuille profile and maximum velocity of $0.0036 \mathrm{~m} / \mathrm{s}$ correspond- 


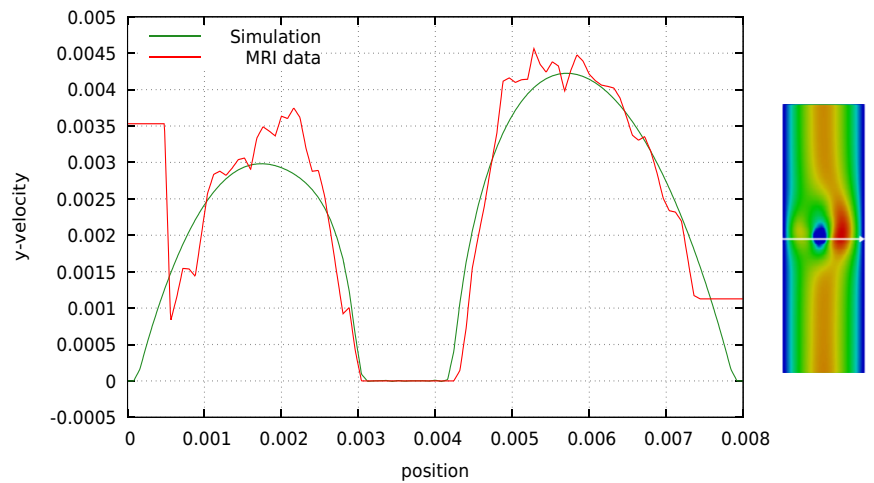

Fig. 7. Velocity profile: $x$-axis. Comparison of velocity profiles along $x$-axis (white line in right figure). The experimental data is shown in red and the simulation result in green. (For interpretation of the references to colour in this figure legend, the reader is referred to the web version of this article.)

ing to the velocity profile of the data (cf. Fig. 2). A pressure condition is used for the outlet and Bounce Back for the remaining shell of the pipe. For the fluid, here isopropanol, a kinematic viscosity of $2.798 \cdot 10^{-6} \mathrm{~m}^{2} / \mathrm{s}$ and density of $786 \mathrm{~kg} / \mathrm{m}^{3}$ is chosen. Further, a relaxation time of $\tau=0.57$ is used. For the adjoint equation the boundary conditions are all set to bounce back (cf. [26]). The design domain, where the control for the optimisation is applied and the object is assumed, is a cylinder with length $0.004 \mathrm{~m}$, radius of $0.002 \mathrm{~m}$ and center at $(0.004 \mathrm{~m}, 0.013 \mathrm{~m}, 0.004 \mathrm{~m})^{T}$, see Fig. 3. The start value for the control is set to $\alpha=-19$ resulting in a lattice porosity of $d_{h}=0.98$. The start value was chosen such that it is sensitive for the optimisation method (cf. [5]). The corresponding permeability can be calculated using (3). The objective domain $\Omega_{J}$ is defined, matching the two-dimensional surface of the MRI data, with $x \in[0.0005 \mathrm{~m}, 0.0075 \mathrm{~m}], y \in[0 \mathrm{~m}, 0.0254 \mathrm{~m}]$ and $z=0.004 \mathrm{~m}$. As the MRI data only contains the $y$ - velocity component, the goal function now reads

$J=\frac{1}{2}\left\|u_{y}^{f}-u_{y}^{*}\right\|_{L^{2}\left(\Omega_{J}\right)}$,

where $u_{y}^{f}$ and $u_{y}^{*}$ are the velocity components in $y$-direction for the simulation and measurement, respectively.

The simulations were carried out on the parallel cluster ForHLR II at Karlsruhe Institute of Technology (KIT).

\subsection{CFD-MRI results}

The challenge here is that the MRI data is only two-dimensional in space and one-dimensional in velocity. Taking a look at the error, here the relative $L 2$ error $\|\cdot\|_{L_{\text {rel }}^{2}}:=\frac{\left\|\mathbf{u}-\mathbf{u}^{*}\right\|_{L^{2}}}{\left\|\mathbf{u}^{*}\right\|_{L^{2}}}$, one can see that the method starts at $41.72 \%$, after only 5 optimisation steps the error is reduced by half and then reaches $11.83 \%$ after 20 steps (cf. Fig. 4). The error stays at around $12 \%$, but as can be seen in Figs. 5-7 the measurement noise is significantly reduced. It can also be seen that the solutions are constrained by the measurement noise. However, the big advantage of the CFD-MRI method is that the solutions now fulfil the porous media BGK-Boltzmann equation, which is not the case for the measurement data. In Fig. 8 the results for the object identification is shown. For this and the following figures a threshold of $d_{h} \leq 0.99$ is used for better visibility. In the background a segment of the MRI data can be seen, and in front a slice of the design domain represented as points showing the lattice porosity. After only 5 steps the basic outline of

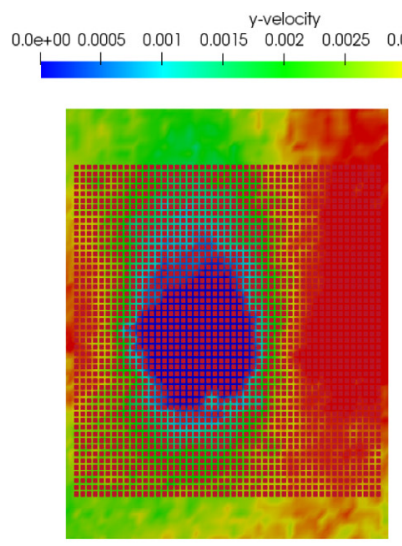

(a) Step 0

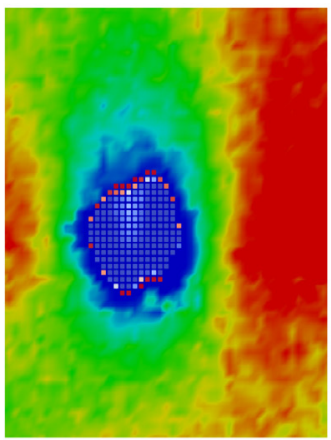

(d) Step 10

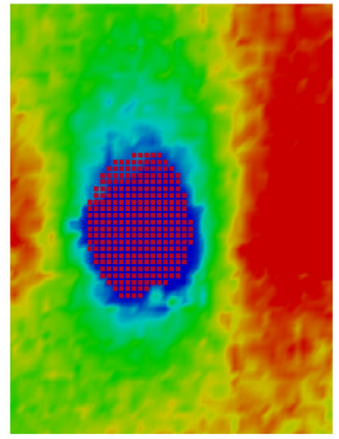

(b) Step 5

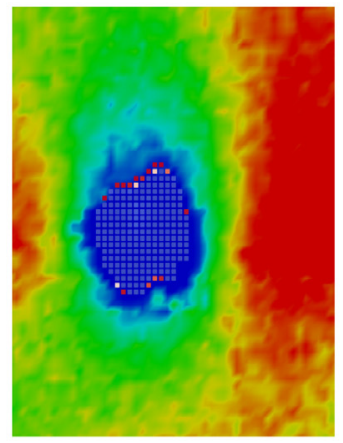

(e) Step 15

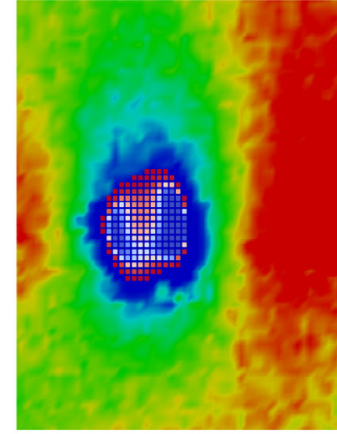

(c) Step 8

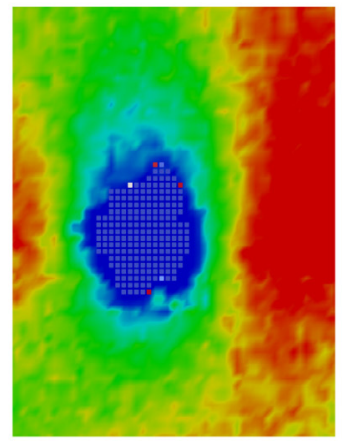

(f) Step 18

Fig. 8. Object identification. Results for the object identification, with the MRI data ( $y$-velocity) in the background and lattice porosity represented as points, where red indicates high and blue low lattice porosity values. The CFD-MRI method locates the cylinder very well in the first 5 steps, but with high lattice porosity. After 18 steps the object has very low lattice porosity, resulting in a solid object. (For interpretation of the references to colour in this figure legend, the reader is referred to the web version of this article.) 


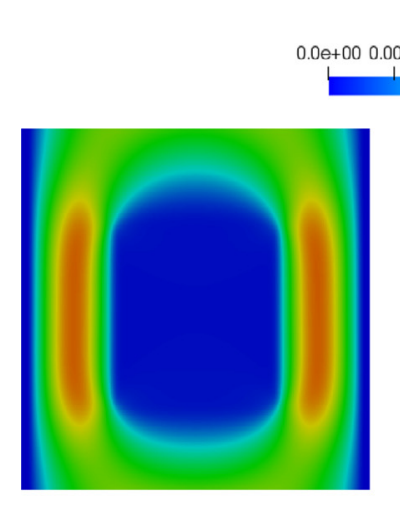

(a) Step 0

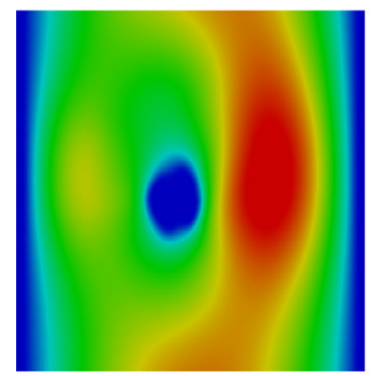

(d) Step 10

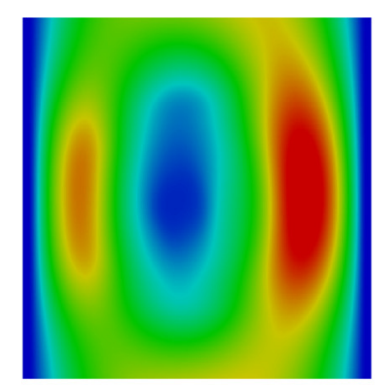

(b) Step 5

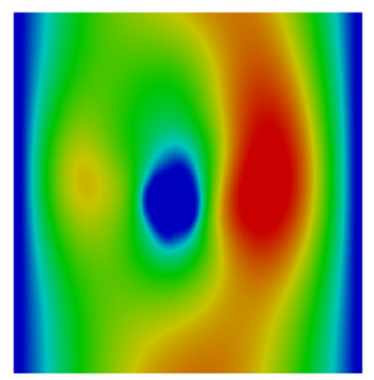

(e) Step 15

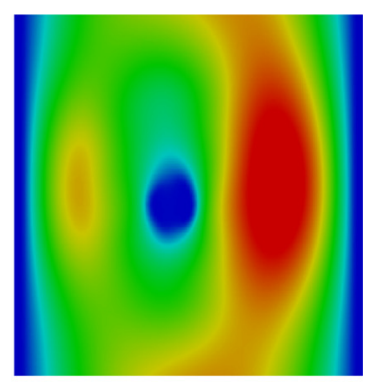

(c) Step 8

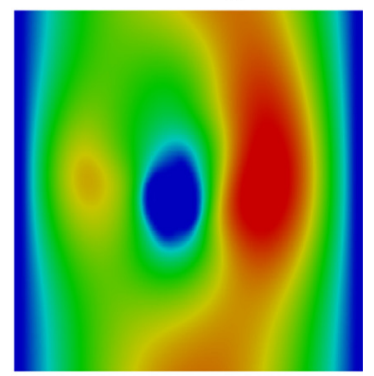

(f) Step 18

Fig. 9. Fluid flow characterisation. Results for the $y$-velocity flow of the simulation for different optimisation step with extraction around the object. The step numbers correspond to those of Fig. 8 .

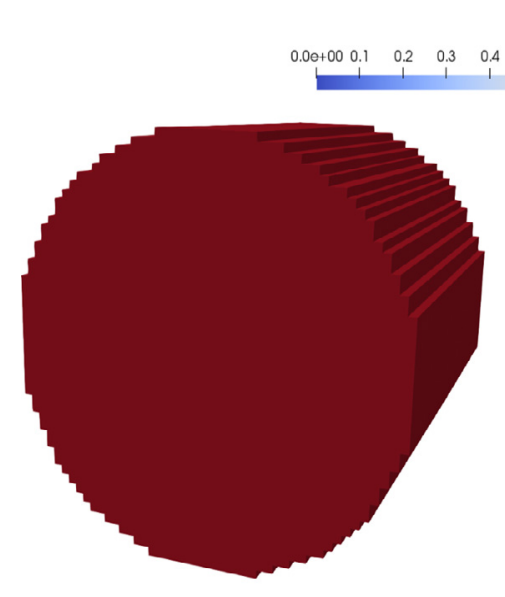

(a) Step 0

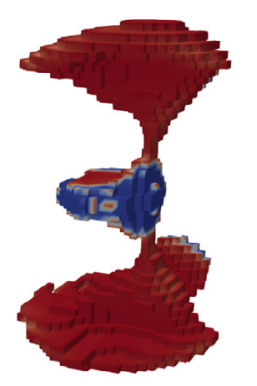

(c) Step 15
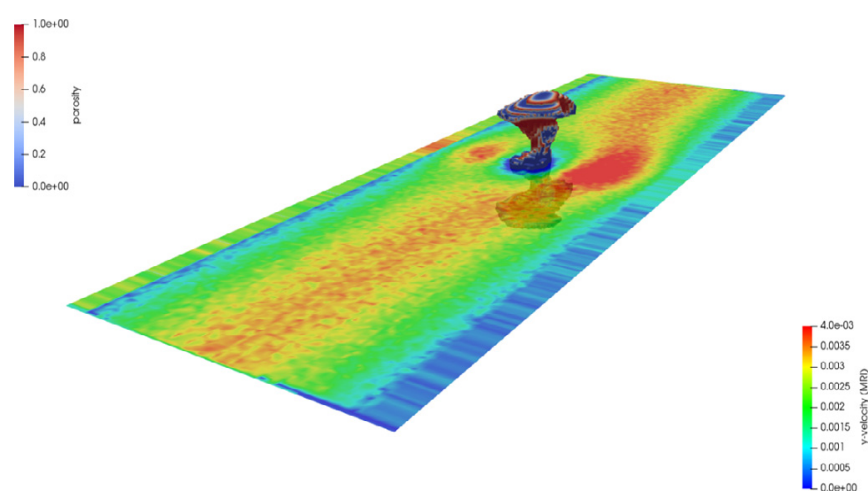

Fig. 11. Object identification. Result of the optimisation algorithm for the cylinder inside the MRI data. Note, that a three-dimensional object is found by only having $2 D$ spatially resolved flow MRI data (plane).

(b) Step 10

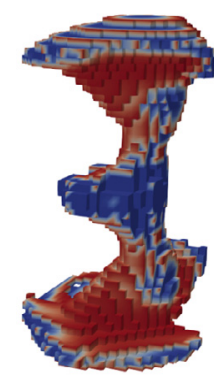

(d) Step 20

the object is found, but with high lattice porosity. After 8 steps the method finds a solid shell, with high lattice porosity interior. The interior gets more solid after 10 steps, with porous outer shell. After 18 steps the interior as well as the outer layer is very solid. Corresponding to the same steps Fig. 9 shows the result for the flow characterisation. It can be seen that the flow is accurately characterised after the object is found. Note that the high start value of $d_{h}=0.98$ is able to stop the flow. This is due to the fact, that $d_{h}$ is not the physical, but the lattice porosity (cf. [5]). Although only $2 D$ spatially resolved data with one velocity component is given, the method finds a three-dimensional object (cf. Fig. 10). At first, the object is only found in the MRI plane, but then becomes more three-dimensional. Note that although the object is not in the middle of the domain, or the objective domain, it is accurately located. Fig. 11 shows the resulting object combined with the MRI data. It can be seen that the two-dimensional data is insufficient to identify the exact three-dimensional object. However, at the same time it shows that the method is not constrained by the dimension of 


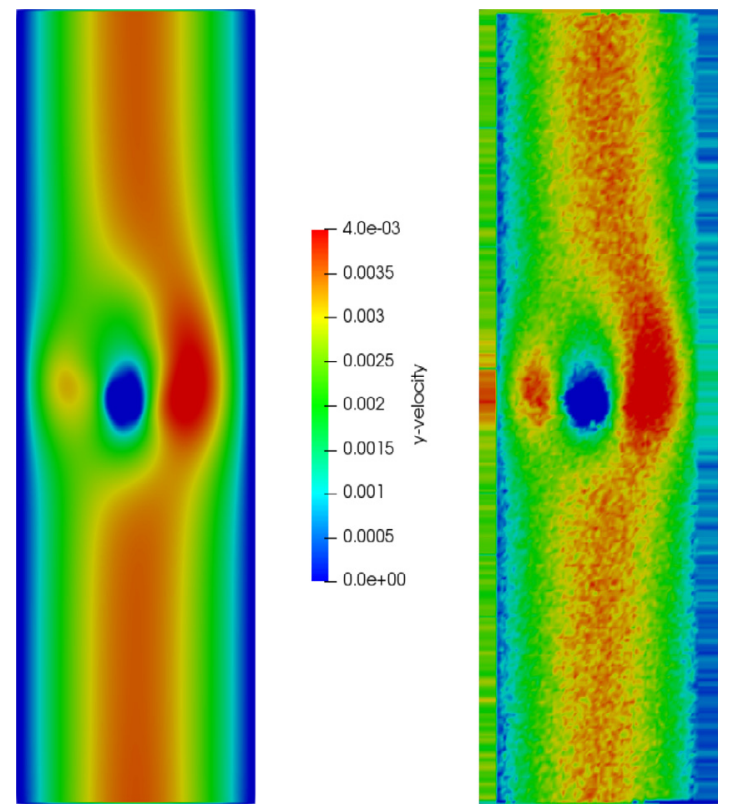

Fig. 12. Comparison. Direct comparison of the CFD-MRI result after 18 steps (left) to the MRI data (right). The fluid flow is accurately characterised with significant reduction of the measurement noise.

the data. Therefore spatially differently oriented data sets can be used within this method. A direct comparison of MRI data and CFD-MRI result can be seen in Fig. 12, which shows a very similar fluid behaviour, but with physical properties and the potential for further analysis in the CFD-MRI result.

\section{Conclusion}

In this article the novel CFD-MRI method for accurate fluid flow characterisation and domain identification was presented. This combination of simulation and measurement data was realised and validated. Thereby a parametrised porous media BGK-Boltzmann equation is used to represent the domain as a porosity distribution. Using the adjoint lattice Boltzmann method for domain identification problems the porosity distribution was changed, such that the fluid field meets the flow MRI data. By this, the CFD-MRI method was able to locate an object and accurately characterise the fluid flow using only $2 D$ spatially resolved MRI data. The measurement noise was thereby significantly reduced with solution that satisfy the physical equations for fluid flow. This results in a powerful tool for the analysis of MRI data.

In the future, noise reduction techniques such as Gaussian filters could be used to reduce noise before or even during simulation. This could combine the advantages of both methods. By smoothing the measurement noise, the optimisation method could find solutions that match the physical models faster. The method should also be extended to characterise porous materials and for calculating various physical quantities like wall shear stresses. The CFD-MRI method could thus help to gain deeper insights into complex applications and underlying physical properties.

\section{Acknowledgements}

The authors would like to thank Willy Dörfler and Hermann Nirschl, both from KIT (Karlsruhe, Germany), for providing the work facilities and excellent supervision. The authors would also like to thank the Steinbuch Centre for Computing at KIT for providing access to their high performance computer ForHLR II, where most computations were carried out as part of the CPE project. The research leading to these results has received funding from the German Research Foundation (DFG 283792184).

\section{References}

[1] Callaghan PT. Principles of nuclear magnetic resonance microscopy. Oxford University Press, New York; 1991. doi:10.1002/ange.19931050256.

[2] Callaghan PT. Translational dynamics and magnetic resonance: principle of pulsed gradient spin echo NMR. Oxford University Press Inc., United States; 2011. doi:10.1080/00107514.2015.1005683.

[3] Kimmich R. NMR - tomography, diffusometry, relaxometry. Springer-Verlag Berlin Heidelberg; 1997. doi:10.1007/978-3-642-60582-6. ISBN 978-3-64264465-8

[4] Krause MJ, Förster B, Mink A, Nirschl H. Towards solving fluid flow domain identification problems with adjoint lattice Boltzmann methods. In: High performance computing in science and engineering '16. Cham: Springer International Publishing; 2016. p. 337-53. ISBN 978-3-319-47066-5. doi:10.1007| 978-3-319-47066-5_23.

[5] Klemens F, Förster B, Dorn M, Thäter G, Krause M]. Solving fluid flow domain identification problems with adjoint lattice Boltzmann methods. Comput Math Appl 2017. Submitted.

[6] Teepakorn C, Grenier D, Fiaty K, Charcosset C. Characterization of hydrodynamics in membrane chromatography devices using magnetic resonance imaging and computational fluid dynamics. Chem Eng Res Des 2016;113:61-73. doi:10.1016/j.cherd.2016.06.027.

[7] Goubergrits L, Riesenkampff E, Yevtushenko P, Schaller J, Kertzscher U, Hennemuth $A$, et al. MRI-based computational fluid dynamics for diagnosis and treatment prediction: clinical validation study in patients with coarctation of aorta. J Magn Reson Imaging 2015;41(4):909-16. doi:10.1002/jmri.24639.

[8] Goubergrits L, Mevert R, Yevtushenko P, Schaller J, Kertzscher U, Meier S, et al. The impact of MRI-based inflow for the hemodynamic evaluation of aortic coarctation. Ann Biomed Eng 2013;41(12):2575-87. doi:10.1007/ s10439-013-0879-2.

[9] Schenkel T, Malve M, Reik M, Markl M, Jung B, Oertel H. MRI-based CFD analysis of flow in a human left ventricle: methodology and application to a healthy heart. Ann Biomed Eng 2009;37(3):503-15. doi:10.1007/s10439-008-9627-4.

[10] Succi S, Foti E, Higuera F. Three-dimensional flows in complex geometries with the lattice Boltzmann method. EPL (Europhys Lett) 1989;10(5):433. doi:10. 1209/0295-5075/10/5/008

[11] Chopard B, Droz M. Cellular automata modeling of physical systems. Cambridge University Press; 1998. doi:10.1017/СB09780511549755.

[12] Chen S, Doolen G. Lattice Boltzmann method for fluid flows. Annu Rev Fluid Mech 1998;30:329-64. doi:10.1146/annurev.fluid.30.1.329.

[13] Sukop MC, Thorne DT. Lattice Boltzmann modeling: an introduction for geoscientists and engineers. Springer; 2006. doi:10.1007/978-3-540-27982-2.

[14] Heuveline V, Krause M, Latt J. Towards a hybrid parallelization of lattice Boltzmann methods. Comput Math Appl 2009;58:1071-80. doi:10.1016/j.camwa. 2009.04.001.

[15] Henn T, Thäter G, Dörfler W, Nirschl H, Krause M. Parallel dilute particulate flow simulations in the human nasal cavity. Comput Fluids 2016;124:197-207. doi:10.1016/j.compfluid.2015.08.002.

[16] Tekitek MM, Bouzidi M, Dubois F, Lallemand P. Adjoint lattice Boltzmann equation for parameter identification. Comput Fluids 2006;35:805-13. doi:10.1016/ j.compfluid.2005.07.015

[17] Liu G, Geier M, Liu Z, Krafczyk M, Chen T. Discrete adjoint sensitivity analysis for fluid flow topology optimization based on the generalized lattice Boltzmann method. Comput Math Appl 2014;68(10):1374-92. doi:10.1016/j.camwa. 2014.09.002.

[18] Nørgaard S, Sigmund O, Lazarov B. Topology optimization of unsteady flow problems using the lattice Boltzmann method. J Comput Phys 2016;307(Supplement C):291-307. doi:10.1016/j.jcp.2015.12.023.

[19] Krause MJ, Thäter G, Heuveline V. Adjoint-based fluid flow control and optimisation with lattice Boltzmann methods. Comput Math Appl 2013;65(6):94560. doi:10.1016/j.camwa.2012.08.007. Mesoscopic Methods in Engineering and Science.

[20] Yaji K, Yamada T, Yoshino M, Matsumoto T, Izui K, Nishiwaki S. Topology optimization using the lattice Boltzmann method incorporating level set boundary expressions. J Comput Phys 2014;274:158-81. doi:10.1016/j.jcp.2014.06.004.

[21] Yaji K, Yamada T, Yoshino M, Matsumoto T, Izui K, Nishiwaki S. Topology optimization in thermal-fluid flow using the lattice Boltzmann method. J Comput Phys 2016;307(Supplement C):355-77. doi:10.1016/j.jcp.2015.12.008.

[22] Pingen G, Evgrafov A, Maute K. Topology optimization of flow domains using the lattice Boltzmann method. Struct Multidiscip Optim 2007;34, 6:507-24. doi:10.1007/s00158-007-0105-7.

[23] Byrd RH, Lu P, Nocedal J, Zhu C. A limited memory algorithm for bound constrained optimization. SIAM J Scient Comput 1995;16(5):1190-208. doi:10. 1137/0916069.

[24] Spaid MAA, Jr Frederick RP. Lattice Boltzmann methods for modeling microscale flow in fibrous porous media. Phys Fluids 1997:9(9):2468-74. doi:10. $1063 / 1.869392$

[25] Krause MJ, Klemens F, Henn T, Trunk R, Nirschl H. Particle flow simulations with homogenised lattice Boltzmann methods. Particuology 2017;34(Supplement C):1-13. doi:10.1016/j.partic.2016.11.001.

[26] Krause MJ. Fluid flow simulation and optimisation with lattice Boltzmann methods on high performance computers - application to the human respiratory system. Karlsruhe Institute of Technology (KIT); 2010. 


\section{Repository KITopen}

Dies ist ein Postprint/begutachtetes Manuskript.

Empfohlene Zitierung:

Klemens, F.; Schuhmann, S.; Guthausen, G.; Thäter, G.; Krause, M. J.

CFD-MRI : A coupled measurement and simulation approach for accurate fluid flow characterisation and domain identification.

2018. Computers \& fluids, 166

doi: $\underline{10.5445 / / R / 1000080936}$

Zitierung der Originalveröffentlichung:

Klemens, F.; Schuhmann, S.; Guthausen, G.; Thäter, G.; Krause, M. J.

CFD-MRI : A coupled measurement and simulation approach for accurate fluid flow characterisation and domain identification.

2018. Computers \& fluids, 166, 218-224.

doi:10.1016/j.compfluid.2018.02.022 